\title{
Nonlinear response of a linear chain to weak driving
}

\author{
D. Hennig, ${ }^{1}$ C. Mulhern, ${ }^{2}$ A. D. Burbanks, ${ }^{1}$ and L. Schimansky-Geier ${ }^{3}$ \\ ${ }^{1}$ Department of Mathematics, University of Portsmouth, Portsmouth PO1 3HF, United Kingdom \\ ${ }^{2}$ Max Planck Institute for the Physics of Complex Systems, Nöthnitzer Strasse 38, 01187 Dresden, Germany \\ ${ }^{3}$ Institut für Physik, Humboldt-Universität zu Berlin, Newtonstraße 15, 12489 Berlin, Germany
}

(Received 20 November 2013; published 8 January 2014)

\begin{abstract}
We study the escape of a chain of coupled units over the barrier of a metastable potential. It is demonstrated that a very weak external driving field with a suitably chosen frequency suffices to accomplish speedy escape. The latter requires passage through a transition state, the formation of which is triggered by permanent feeding of energy from a phonon background into humps of localized energy and elastic interaction of the arising breather solutions. In fact, cooperativity between the units of the chain entailing coordinated energy transfer is shown to be crucial for enhancing the rate of escape in an extremely effective and low-energy cost way where the effects of entropic localization and breather coalescence conspire.
\end{abstract}

DOI: 10.1103/PhysRevE.89.012906

Recently, there has been increasing interest in the escape of coupled degrees of freedom or chains of interacting units out of metastable states [1-7]. Escape is accomplished when the considered object overcomes a potential barrier separating the local minimum of the potential landscape from a neighboring domain of attraction. The activation energy required to surmount the energetic bottleneck can be provided in different ways. There is the possibility of stochastic escape occurring in the presence of a heat bath that is sampled for the optimal fluctuations triggering an event of escape. Alternatively, in the noise-free situation the energy can be supplied in a single shot under microcanonical circumstances. Spontaneous localization of energy due to modulational instability promotes the formation of localized humps on the chain. Escape is connected with the crossing of a saddle point in configuration space. In particular, a sufficient amount of energy needs to be concentrated in an associated critical nucleus called the transition state. Adopting the latter and by passing through it, the chain is able to surmount the barrier. In addition, the activation energy may be injected into the system by an external time-dependent field [8-11] and waves [12]. The objective of this paper is to elaborate on an escape scenario of a chain of interacting units over the barrier of a metastable potential promoted by a very weak external periodic field. The initially almost homogeneous chain, possessing virtually no energy, is brought into the nonlinear regime where it may exhibit spontaneous energy localization. This process is driven by an instability of the homogeneous chain with respect to spatial fluctuations triggered by the external field. Subsequently, a localized pattern may form on the chain. With this work, we intend to demonstrate that already a very weak forcing with a suitably chosen frequency suffices so that the necessary energy gets localized in order that the chain locally adopts and overcomes the transition state promoting escape over the barrier.

We study a one-dimensional lattice of nonlinear and driven coupled oscillators. Throughout the following, we use dimensionless parameters, as obtained after appropriate scaling of the corresponding physical quantities. The coordinate $q$ of each individual nonlinear oscillator with a unit mass evolves in a cubic, single-well on-site potential of the form

$$
U(q)=\frac{\omega_{0}^{2}}{2} q^{2}-\frac{a}{3} q^{3}, \quad a \geqslant 0 .
$$

PACS number(s): 05.45.-a, 63.20.Pw, 63.20.Ry

This potential possesses a metastable equilibrium at $q_{\min }=$ 0 , corresponding to the rest energy $E_{\min }=0$ and exhibits a maximum that is located at $q_{\max }=\omega_{0}^{2} / a$ with energy $E_{\max } \equiv$ $\Delta E=\omega_{0}^{6} /\left(6 a^{2}\right)$. Thus, in order for particles to escape from the potential well of depth $\Delta E$ over the energy barrier and subsequently into the range $q>q_{\max }$, a sufficient amount of energy needs to be supplied. The lattice dynamics is governed by the following system of coupled equations:

$$
\ddot{q}_{n}+\omega_{0}^{2} q_{n}-a q_{n}^{2}-\kappa\left[q_{n+1}+q_{n-1}-2 q_{n}\right]-F(t)=0 .
$$

The coordinates $q_{n}(t)$ quantify the displacement of the oscillator in the local on-site potential $U$ at lattice site $n \in[1, N]$. The oscillators, referred to as units, are coupled linearly to their neighbors with interaction strength $\kappa$. A homogeneous external modulation field $F(t)$ acts globally upon the system. The field is provided by a periodic monochromatic driving force of amplitude $A$, frequency $\omega$, and phase $\theta_{0}$ given as

$$
F(t)=A \sin \left(\omega t+\theta_{0}\right) .
$$

We use periodic boundary conditions according to $q_{N+1}=$ $q_{1}$ and fix the parameters as follows: $\omega_{0}^{2}=2, \theta_{0}=0$, and $a=1$, yielding $\Delta E=4 / 3$. In what follows, we apply a very weak external periodic field of small amplitude $A=0.003$ which contributes with a value of $\min _{t} F(t) q_{\max }=-A q_{\max }=$ -0.006 to a diminutive lowering of the potential barrier only. A deterministic escape scenario in the conservative, undriven limit of system (2) has been explored in Ref. [7]. The system (2) has been integrated numerically using a fourth-order Runge-Kutta scheme. In our simulations, the chain consists of $N=100$ units. The units are initialized such that the positions of all units are randomized around the bottom of the potential $q_{0}=0$ in the range $\left|q_{n}(0)-q_{0}\right|<0.1$ so that the energy of a unit does not exceed the value $E_{0}=0.01=$ $0.0075 \Delta E$ and hence is negligibly small compared to the barrier energy $\Delta E$. The whole chain is thus initialized close to an almost homogeneous state, but yet sufficiently displaced in order to generate nonvanishing interactions, enabling the exchange of energy among the coupled units. Starting from a random pattern with units containing vanishingly little energy compared to the barrier energy, the question then is, what is the impact of the very weak periodic driving force regarding escape of the chain over the barrier? As far as the choice of 
the frequency of the external periodic field $\omega$ is concerned, a value close to the frequency of harmonic oscillations of the units close to the bottom of the potential well, $\omega_{0}=\sqrt{2}$, seems appropriate since for a start phonons need to be excited on the chain. The band of phonon frequencies $\omega$ is determined by

$$
\omega_{\mathrm{ph}}^{2}=\omega_{0}^{2}+4 \kappa \sin ^{2}\left(\frac{k}{2}\right),
$$

with wave numbers $k \in[0, \pi]$. The breathers emerging on the chain have their frequencies below the lower edge of the phonon band.

For a driving frequency $\omega=1.4 \simeq \sqrt{2}$ the chain rapidly attains a nonequilibrium steady state of almost uniform amplitude, $q_{n}(t)=q_{0}(t)$, to which it remains entrained for some time. This state is in very good agreement with the solution obtained from the linear system, which for zero initial conditions $q_{n}(0)=\dot{q}_{n}(0)=0$ is given by

$$
q_{n}(t)=q_{0}(t)=\frac{A \omega}{\omega_{0}^{2}-\omega^{2}}\left[\frac{1}{\omega} \sin (\omega t)-\frac{1}{\omega_{0}} \sin \left(\omega_{0} t\right)\right] .
$$

Using addition theorems, we can express the solution $q_{0}(t)$ for $\omega_{0} \approx \omega$ as

$$
q_{0}(t) \simeq \frac{2 A}{\omega_{0}^{2}-\omega^{2}} \cos \left(\frac{\omega+\omega_{0}}{2} t\right) \sin \left(\frac{\omega-\omega_{0}}{2} t\right) .
$$

The (slowly varying) envelope of $q_{0}(t)$ attains its maximal amplitude at $t=\pi /\left(\omega-\omega_{0}\right)$. At this moment, despite the fact that the amplitude of the chain has grown already (to the brink of the weakly anharmonic regime), the amount of gained energy remains too small to raise the chain near the potential barrier. Nevertheless, as the chain is initialized in a nonhomogeneous state (albeit of low amplitude), the units perform small-scale fluctuations around the nonequilibrium uniform steady state giving the possibility to render the latter unstable. For nearresonance driving, $\omega \simeq \omega_{0}$, we adopt the parameter values such that $v_{0}=2 A /\left(\omega_{0}^{2}-\omega^{2}\right) \lesssim 1$, bounding accordingly the amplitude of $q_{0}(t) \simeq v_{0} \cos \left[0.5\left(\omega+\omega_{0}\right) t\right] \sin \left[0.5\left(\omega-\omega_{0}\right) t\right]$. While the chain retains the nonequilibrium steady-state solution $q_{0}(t)$ with some more growth of its amplitude, the weakly nonlinear regime is entered (see also below). In the following, we perform a stability analysis of the uniform nonequilibrium steady-state solution $q_{0}(t)$. Toward that end, we write with respect to spatial perturbations $u_{n}(t)$,

$$
q_{n}(t)=q_{0}(t)+u_{n}(t) .
$$

For the imposed periodic boundary conditions, we express the perturbations as a Fourier-series expansion,

$$
u_{n}(t)=\sum_{k} \xi_{k}(t) \exp (i k n)+\text { c.c. }
$$

with wave numbers $k \in[0, \pi]$ yielding a Mathieu-type equation for the mode amplitudes $\xi_{k}$,

$$
\ddot{\xi}_{k}+\left\{\omega_{\mathrm{ph}}^{2}-2 a u_{0} \cos \left(\frac{\omega+\omega_{0}}{2} t\right) \sin \left(\frac{\omega-\omega_{0}}{2} t\right)\right\} \xi_{k}=0,
$$

where $\omega_{\text {ph }}$ is given in Eq. (4) and $u_{0}$ is the amplitude of the nonequilibrium steady-state solution in the weakly nonlinear regime.

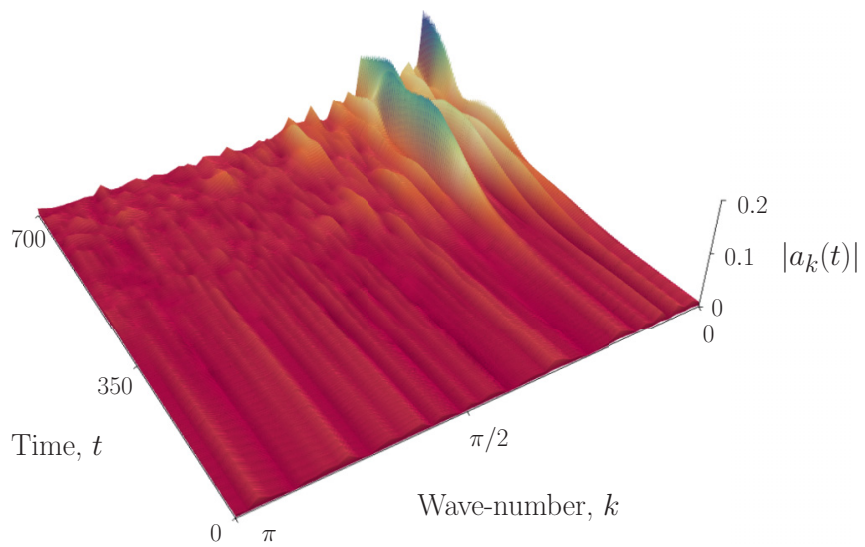

FIG. 1. (Color online) Time evolution of the spatial Fourier transform of $q_{n}(t)$. The values of the parameters are $\omega_{0}^{2}=2, a=1$, $A=0.003, \omega=1.4, \theta_{0}=0$, and $\kappa=0.1$.

We investigated the instability region of this Mathieu-type equation in the $A-k$ parameter plane for driving frequency $\omega=1.4$. For $A=0.003$, the position of the bottom of the instability band is at $k_{c} \simeq 0.16$ determining the critical unstable wave number. Thus the creation of a pattern consisting of localized humps (breathers), with wavelength determined by $\lambda_{c}=2 \pi / k_{c}$, can be expected. In fact, the time evolution of the spatial Fourier transform,

$$
q_{n}(t)=\sum_{k} a_{k}(t) \exp (i k n)+\text { c.c., }
$$

displayed [for one realization of frozen noise $\left|q_{n}(0)-q_{0}\right|<$ 0.1 and $\left.p_{n}(0)=0\right]$ in Fig. 1, corroborates this feature. For times $t \gtrsim 400$ (corresponding to 90 periods $T_{0}=2 \pi / \omega_{0}$ of harmonic oscillations near the bottom of the potential well), the chain departs from the regime of nearly uniform steady state, which is indicated in the spatial Fourier spectrum by the formation of pronounced Fourier components in the domain of low wave numbers.

In accordance with our stability analysis, one notices for the spatiotemporal evolution of the coordinates $q_{n}(t)$ that due to the instability of the steady state, and the ensuing energy exchange among the units, after some time small fractions of energy can become localized in humps (breathers). We observe that a pattern evolves over the course of time where for some lattice sites the amplitudes grow considerably while remaining small in the adjacent regions. That is, an array of large-wavelength (small-wave-number) chaotic breathers is formed. Upon moving, these breathers tend to collide inelastically with others. In fact, various breathers merge to form larger-amplitude breathers, proceeding preferably such that the larger-amplitude breathers grow at the expense of the smaller ones. As a result, more energy becomes strongly concentrated within confined regions of the chain. If at least one of the breathers can be sufficiently strongly amplified on a segment of the lattice such that the associated maximal amplitude grows to the proximity of the barrier level, then a crossing of the energy barrier for this segment becomes achievable. Moreover, in Ref. [7] it was shown that such a localized state might adopt the hairpin shape of the critical localized mode (transition state), and if the involved 


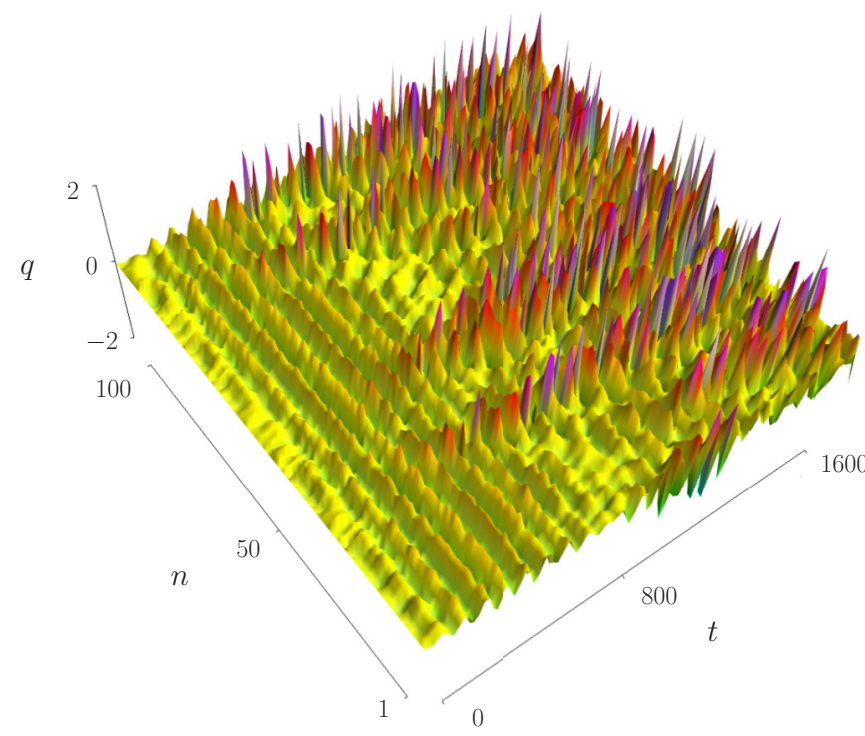

FIG. 2. (Color online) Spatiotemporal pattern of the coordinates $q_{n}(t)$ prior to escape for very weak periodic driving. The values of the parameters are $\omega_{0}^{2}=2, a=1, A=0.003, \omega=1.4, \theta_{0}=0$, and $\kappa=0.1$. The pattern is shown up to times shortly before the escape of the chain takes place.

amplitudes become overcritical, escape is realized. However, for this to happen, at first one unit has to absorb sufficient energy to completely surmount the barrier. For our choice of parameter values, the energy of the transition state amounts to 1.35 $\Delta E$ [7]. We emphasize that it is the comparatively high mobility of the chaotic breathers leading to collisions with other (standing and/or moving) breathers that enhances the concentration of energy.

In Fig. 2, we show a typical spatiotemporal pattern $q_{n}(t)$ for a very weakly driven chain for the same realization of frozen noise $\left|q_{n}(0)-q_{0}\right|<0.1$ and $p_{n}(0)=0$ as in Fig. 1. A few moving localized humps (chaotic breathers) are discernible. After $\sim 1690$ time units, the chain adapts locally a hairpinlike configuration, and by passing through the latter with sufficiently large (positive) velocities, a subsequent escape of the involved units is initiated. Return of the escaped units over the barrier into the original potential well is virtually excluded, and consecutively all units, being pulled by the already escaped ones, manage to climb over the barrier. Eventually the chain propagates freely beyond the potential barrier with increasing kinetic energy. We emphasize that the global escape of the chain relies on appropriate cooperations of the chain units leading to enhanced energy concentrationan effect that is absent for uncoupled units. In fact, for vanishing coupling $\kappa=0$ none of the periodically driven units escaped during the long simulation time $T_{s}=10^{5} \sim$ $2252 T_{0}=2 \pi / \omega_{0}$.

To quantify the change of the energy of the chain in response to the external driving and internal processes of energy redistribution, we attribute to each unit a site energy

$$
E_{n}=E_{\mathrm{kin}, n}+E_{\mathrm{pot}, n}+E_{\mathrm{driv}, n}
$$

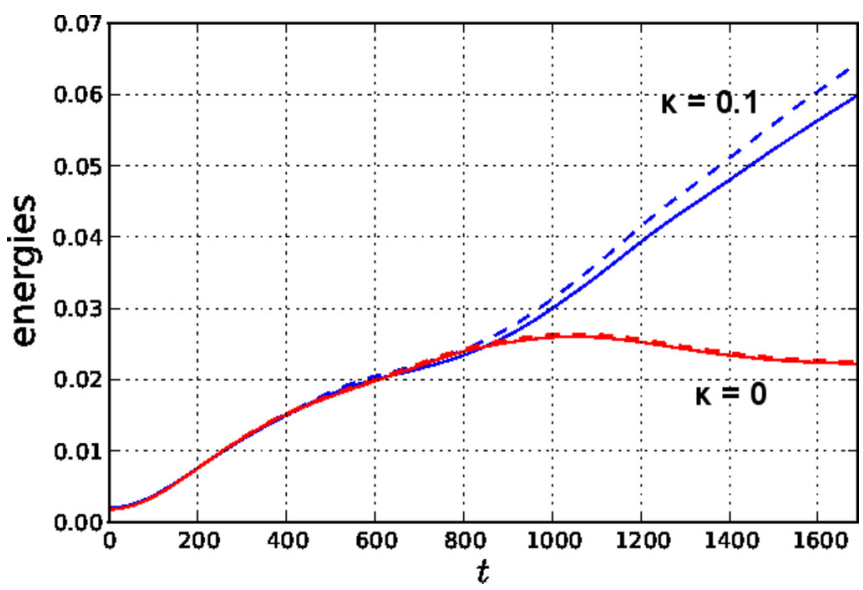

FIG. 3. (Color online) Temporal evolution of the average kinetic (solid line) and average potential (dashed line) energy for weak periodic driving for coupled units $(\kappa=0.1)$ and uncoupled units $(\kappa=0)$ as indicated in the plot. The values of the remaining parameters are $\omega_{0}^{2}=2, a=1, A=0.003, \omega=1.4$, and $\theta_{0}=0$. The time evolution is shown up to times shortly before the escape of the chain takes place.

where the kinetic site energy, the potential site energy, and the driving energy are given by

$$
\begin{gathered}
E_{\mathrm{kin}, n}=\frac{1}{2} \dot{q}_{n}^{2}, \\
E_{\mathrm{pot}, n}=U\left(q_{n}\right)+\frac{\kappa}{4}\left(q_{n+1}-q_{n}\right)^{2}+\frac{\kappa}{4}\left(q_{n}-q_{n-1}\right)^{2}, \\
E_{\mathrm{driv}, n}=F(t) q_{n},
\end{gathered}
$$

respectively, and we compute the average kinetic energy and the average potential energy of the chain defined as

$$
\begin{aligned}
& \bar{E}_{\mathrm{kin}}=\frac{1}{N} \sum_{n=1}^{N} \frac{1}{T} \int_{0}^{T} E_{\mathrm{kin}, n}(t) d t, \\
& \bar{E}_{\mathrm{pot}}=\frac{1}{N} \sum_{n=1}^{N} \frac{1}{T} \int_{0}^{T} E_{\mathrm{pot}, n}(t) d t .
\end{aligned}
$$

Due to its diminutive size, the contribution of the driving energy $E_{\text {driv }}$ can be ignored.

Figure 3 shows the temporal evolution of $\bar{E}_{\text {kin }}$ and $\bar{E}_{\text {pot }}$ contained in the chain of coupled units. Throughout the time, the chain of coupled units gains on average kinetic and potential energy from the external driving field. Up to times $t \lesssim 400$, when the chain is still in the nearly uniform nonequilibrium steady state, the average potential and kinetic energy are of equal amount. For later times, when the first humps form on the chain, the dynamics enters the weakly nonlinear regime coinciding with the fact that the ratio of the anharmonic part and the harmonic part of the potential exceeds a threshold value, viz.

$$
\frac{\frac{a}{3} \sum_{n}\left|q_{n}\right|^{3}}{\frac{\omega_{0}^{2}}{2} \sum_{n} q_{n}^{2}}>0.1 .
$$


As a hallmark of soft potentials, the energy is on average not equally divided between potential and kinetic ones, as for a soft oscillator the oscillation frequency decreases and the density of states increases with increasing amplitude (energy). That is, on average the energy spends relatively more time in potential form than in kinetic form. For comparison we show also the average energies for uncoupled units, viz. for $\kappa=0$, starting with the same initial conditions as the coupled units. Until $t \simeq 400$ the behavior of coupled units and uncoupled units is virtually the same. However, for times $t \gtrsim 400$ the first minor deviations occur, and later at $t \simeq 850$ the average kinetic and potential energy of coupled units increases further monotonically, while for uncoupled units, near stagnation is found giving evidence that cooperative effects are crucial for the growth of energy observed for coupled units. Concerning the degree of localization, it is illustrative to consider the energy localization parameter

$$
C_{0}(t)=N \frac{\sum_{n=1}^{N} E_{n}^{2}}{\left(\sum_{n=1}^{N} E_{n}\right)^{2}}
$$

introduced in Ref. [13]. Starting from a state of virtually equipartition corresponds to $C_{0}(0) \simeq 1$. Over the course of time $C_{0}(t)$ increases, indicating that the energy becomes localized at a few sites. With further enhancement of localization caused by breather coalescence, $C_{0}(t)$ grows and prior to escape reaches a value of almost $N$, being associated with strong localization of energy at a single site.

In this context, we also consider the average temperature of the chain defined as the mean kinetic energy in a frame comoving with the center of mass of the chain as

$$
T_{\text {chain }}=\frac{1}{2}\left\langle\left[\dot{q}_{n}(t)-\overline{\dot{q}}(t)\right]^{2}\right\rangle,
$$

where $\overline{\dot{q}}$ is the velocity of the center of mass. Figure 4 shows the temporal behavior of the system temperature up to the moment when the first unit surmounts the potential barrier initiating global escape. Comparing the behaviour of the average kinetic energy $\bar{E}_{\text {kin }}$ and the temperature of the chain $T_{\text {chain }}$, it becomes clear that the latter follows the former, $T \simeq(0.5-0.8) \bar{E}_{\mathrm{kin}}$,

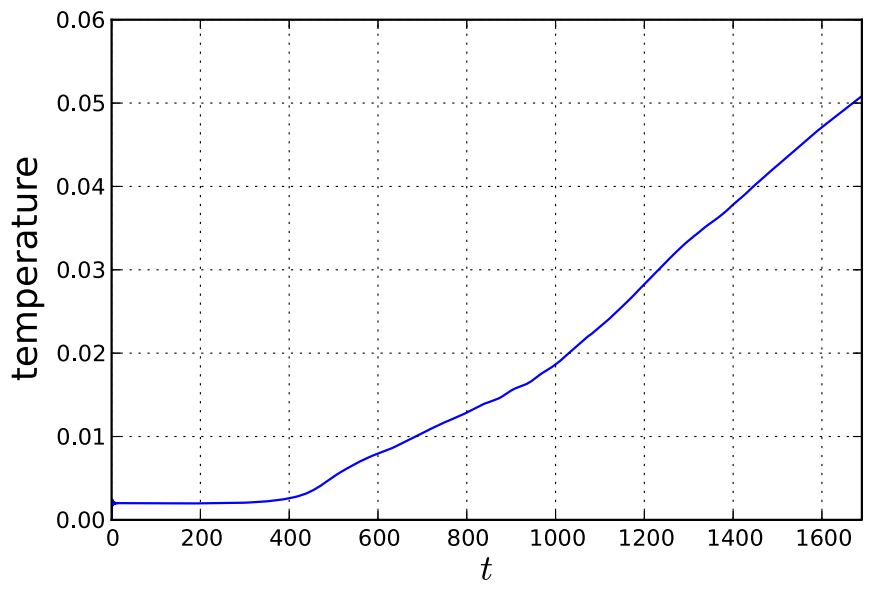

FIG. 4. (Color online) Temperature of the chain for weak periodic driving. The values of the parameters are $\omega_{0}^{2}=2, a=1$, $A=0.003, \omega=1.4, \theta_{0}=0$, and $\kappa=0.1$. The time evolution is shown up to times shortly before the escape of the chain takes place. and thus, comparatively, the kinetic energy of the center of mass of the chain diminishes as time progresses.

During the time when the chain is still in the linear regime $(t \lesssim 400)$, the temperature remains constant indicating little variability between the units (see above). Upon entering the nonlinear regime related with the emergence of breathers, the average temperature increases monotonically but with a weakly varying rate. In fact, after 1000 time units (equivalent to $\sim 227$ periods of harmonic oscillations near the bottom of the potential well), the slope of the graph increases further, indicating a regime of enhanced interactions ("collisions") caused by moving chaotic breathers traversing the chain (see below). With further growth of the temperature along with enhanced localization of energy, the chain eventually overcomes the potential barrier. The temperature exhibits a stretched exponential time dependence

$$
T_{\text {chain }} \sim \exp \left(a t^{b}\right)
$$

with parameter values $a \simeq 0.01$ and $b \simeq 0.75$. Analogous behavior was found in the reverse problem concerning the relaxation dynamics of a lattice chain which is initially thermalized and afterward put in contact with a cold bath $[14,15]$.

To gain more insight into the nature of the escape process, we consider the rate of change of the site energy,

$$
\begin{aligned}
\dot{E}_{n}= & \frac{\kappa}{2}\left[\left(q_{n+1}-q_{n}\right)\left(\dot{q}_{n+1}-\dot{q}_{n}\right)-\left(q_{n}-q_{n-1}\right)\left(\dot{q}_{n}-\dot{q}_{n-1}\right)\right] \\
& +F(t) \dot{q}_{n}+\dot{F}(t) q_{n} .
\end{aligned}
$$

The energy of a unit can be changed via two channels. There is the possibility of energy exchange with the external field $F(t)$ involving both the velocities $\dot{q}_{n}$ and coordinates $q_{n}$ of a unit. With proper phase relations between the oscillations of a coordinate $q_{n}(t)$ and the derivative of the external periodic field $\dot{F}(t)$ and/or between the velocity $\dot{q}_{n}(t)$ and the external periodic field $F(t)$, a unit can gain energy during an interval $\Delta t$ providing $\int_{t}^{t+\Delta t} d \tau\left[\dot{q}_{n}(\tau) F(\tau)+q_{n}(\tau) \dot{F}(\tau)\right]>0$. In particular, during the initial stage of linear behavior of the system, regardless of whether it is coupled or uncoupled, its energy gain from the external field is fairly pronounced. This behavior is characterized by nearly in-phase motion of the derivative of the external field $\dot{F}(t)$ and the mean value of the coordinates $\bar{q}$ whose evolution is determined by Eq. (5) as well as in-phase motion of the mean value of the velocities $\overline{\dot{q}}(t)$ and the external field $F(t)$.

Upon entering the anharmonic regime for coupled units, a pattern of localized humps arises, which naturally is impossible for noncoupled units. The question is, what is the mechanism of ongoing energy injection into breathers being responsible for their observed growth? With the onset of spatiotemporal chaos, the almost in-phase coherence between $\bar{q}$ and $\dot{F}(t)$ on the one hand, and between $\bar{q}$ and $F(t)$ on the other, can get temporarily and/or locally lost impeding further energy growth. Nonetheless, there are periods during which the phase correlations are maintained so that (at least locally) energy pumping from the external field into the corresponding units of the chain is accomplishable.

In addition, the permanent impact of the external periodic field is that itinerant chaotic breathers merge eventually 


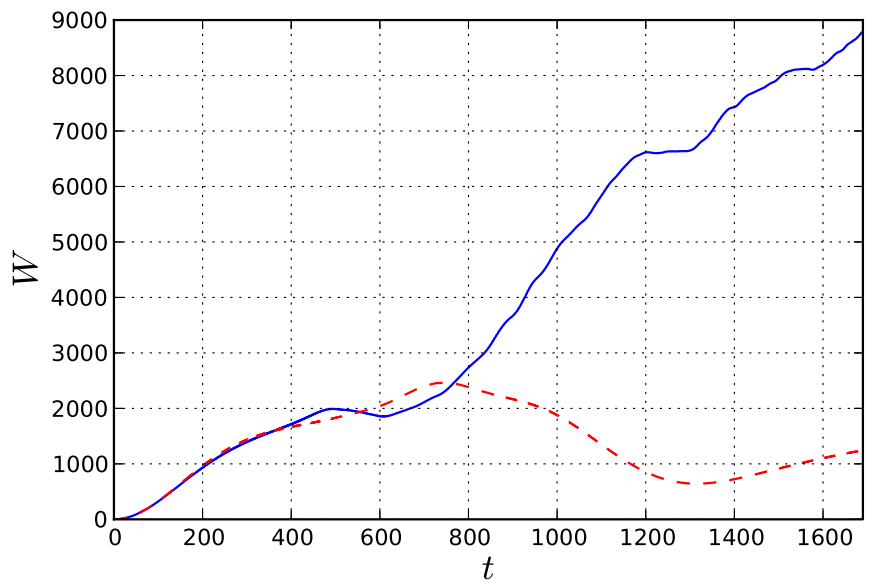

FIG. 5. (Color online) Work done on the chain by the weak periodic driving for $\kappa=0$ (dashed line) and $\kappa=0.1$ (solid line). The values of the remaining parameters are $\omega_{0}^{2}=2, a=1, A=0.003$, $\omega=1.4$, and $\theta_{0}=0$.

with large-amplitude breathers. In fact, in between the few large-amplitude standing breathers, the external driving, with frequency almost coinciding with the frequency at the lower edge of the phonon band, creates permanently a phonon "bath" background feeding energy into arising small-amplitude (chaotic) breathers. We emphasize that the cooperativity of the units plays a vital role in achieving escape in such an effective and low-energy cost manner. To gain more insight into the energy gain of the coupled chain, we plot in Fig. 5 the work done by the external field on the chain, which is determined by

$$
W(t)=\frac{1}{N} \sum_{n=1}^{N} \int_{0}^{t} F(\tau) \dot{q}_{n}(\tau) d \tau .
$$

During the initial linear regime, characterized by almost in-phase motion of the external field $F(t)$ and the mean value of the velocities $\bar{q}$, the work done on the uncoupled units and the coupled ones coincides. However, at later times the difference becomes drastic as the work done on the coupled units increases over the course of time, whereas the work done on the uncoupled units is subdued and even diminishes temporarily. For the former, this means that on average the velocities of the units not involved in standing large-amplitude breathers enhance in conjunction with the external periodic field. This instigates motion of small-amplitude chaotic breathers (emanating from the phonon background) along the chain, and the energy of the latter gets absorbed by standing higheramplitude breathers upon coalescence. Furthermore, as long as the standing breathers are of undercritical amplitude, and hence their frequency remains close to the driving frequency, direct (resonant) injection of energy from the external field into them is possible, as a result of which the amplitude of the latter grows and its frequency gets shifted further below the phonon band. Eventually for overcritical amplitudes the resulting mismatch between the frequency of the breathers and the frequency of the external field becomes too great for further direct energy feeding from the external field into the breather. Then the only way for a breather to gain more energy is by means of internal energy distribution between the units of the chain.

The internal mechanism of energy exchange along the chain is due to the coupling term being responsible for the energy exchange of a unit with its nearest neighbors via the springs connecting the units. The larger the stress $\left|q_{n \pm 1}-q_{n}\right|$ - the potential energy stored in the spring-and/or the velocities $\left|\dot{q}_{n \pm 1}\right|,\left|\dot{q}_{n}\right|$, the higher is the rate of internal change of the site energy. The internal exchange of energy between neighboring units is blocked when $q_{n \pm 1}=q_{n}$ or $\dot{q}_{n \pm 1}=-\dot{q}_{n}$, that is, when neighboring oscillators perform equal amplitude in-phase motion or out-of-phase motion with respect to each other with opposite sign of velocities. Moreover, once a unit has acquired a high energy, it is retained for a fairly long time due to the fact that in a soft oscillator the energy spends relatively more time in potential than in kinetic form. The reason for this is that in soft potentials the oscillation frequency decreases with increasing amplitude. In conjunction with the fact that the density of states increases with increasing amplitude, attaining and preserving higher amplitudes is entropically more favorable (see also [16]). Thus, during the major part of an oscillation period of a unit, after it has gained energy from the external field, its neighbors, or impacting moving breathers, the displacement of this unit remains large while the velocity is low. Therefore, this entropic localization mechanism impedes the energy exchange of a higher-amplitude unit with the surroundings. Conclusively, localization of energy minimizes the free energy as it is favored, with respect to maximization of entropy, that the energy gaining units populate regions in phase space where the density of states is higher.

Finally, we present our results regarding the mean escape time $T_{\text {esc }}$ of the chain. The escape time for the chain is defined as the average of the moments at which the $N$ amplitudes of the escaping units pass the value $q=200=100 q_{\max }$ beyond the barrier location. $T_{\text {esc }}$ versus the driving frequency for a small driving amplitude $A=0.003$ is displayed in Fig. 6. The averages were performed over 100 realizations of random initial conditions. There is a window of frequencies $1.395 \lesssim \omega \lesssim 1.437$ for which speedy escape is accomplished,

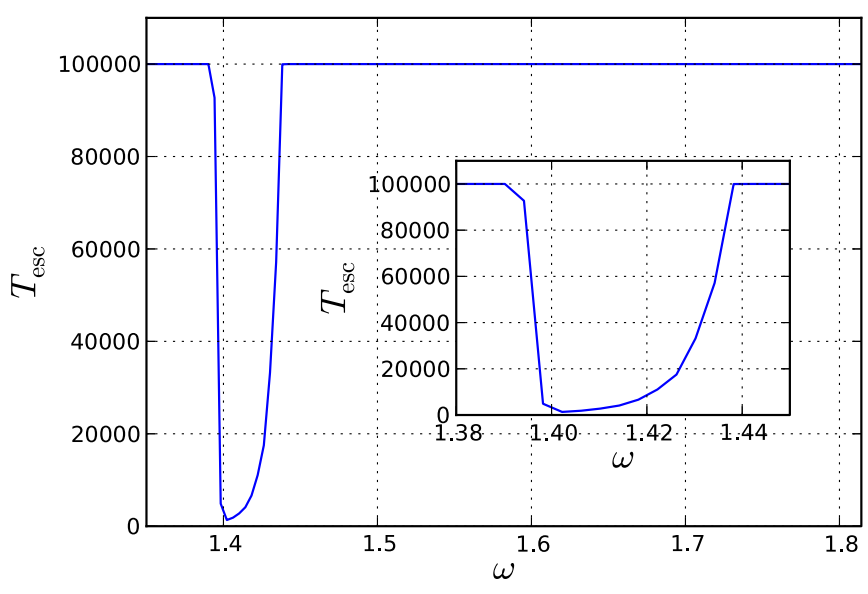

FIG. 6. (Color online) Mean escape time of the chain as a function of the driving frequency $\omega$ for a small fixed driving strength $A=0.003$. The values of the remaining parameters are $\omega_{0}^{2}=2$, $a=1, A=0.003, \theta_{0}=0$, and $\kappa=0.1$. 
and outside of this window not a single event of escape takes place throughout the simulation time $T_{s}=10^{5}$. The window of the driving frequencies associated with speedy escape shown in Fig. 6 has an overlap with the phonon band penetrating the latter from its lower edge, underlining the fact that permanently impinging phonons (arising as the result of driving the system within this frequency range) are paramount for the creation of breathers promoting eventually the escape process. These breathers have frequencies contained in the window of speedy escape just below the phonon band.

In summary, in this study we have investigated the escape problem of a chain of harmonically coupled units over the barrier of a metastable potential. Energy is injected into the system by means of an applied external time-periodic field. Notably, even for a very weak driving force we have observed fast escape for a chain situated initially extremely close to the bottom of the potential well and thus containing a vanishingly small amount of energy. For a suitably chosen driving frequency almost coinciding with the frequency at the lower edge of the phonon band of linear oscillations, as a start, an almost uniform oscillating state of the chain is excited. The amplitude of the latter rises (slowly) in time, and upon entering the weakly nonlinear regime the almost uniform state becomes unstable with respect to spatial perturbations. This triggers the formation of a few localized humps (standing breathers) coexisting with a phonon "bath" background in between them. Due to the effect of entropic localization for the standing breathers, their energy-reduction process is impeded. Contrarily, the process in the other direction is entropically favored. That is, due to the fact that the driving frequency lies just below the phonon band, further resonant energy pumping by the external field into standing breathers is possible, provided a proper phase relation is retained between them. In fact, the associated growth of the amplitude of the breathers enhances even entropic localization. However, as with growing amplitude, the frequency of a breather diminishes, and there results a frequency mismatch between the external field and the standing large-amplitude breather hampering direct substantial energy feeding from the external field into it. Therefore, at this stage the only way a breather can gain more energy is by processes of internal energy redistribution along the chain. Conclusively, choosing the frequency of the external driving just below the phonon band is advantageous for two reasons: First, emerging standing breathers can become amplified by direct energy gain from the (almost) resonant external field. At the same time, externally driving with a frequency almost equal to that of harmonic oscillations near the bottom of the potential well generates permanently a phonon "bath" background between the standing breathers forming the source for the emergence of mobile chaotic breathers. The mergence of the itinerant chaotic breathers with standing breathers contributes to their growth. Eventually for overcritical amplitudes a standing breather adopts the shape and energy content of the transition state, and by passing through the latter, escape of the chain over the barrier gets instigated.
[1] W. Sung and P. J. Park, Phys. Rev. Lett. 77, 783 (1996).

[2] P. J. Park and W. Sung, Phys. Rev. E 57, 730 (1998); J. Chem. Phys. 108, 3013 (1998); 111, 5259 (1999).

[3] I. E. Dikshtein, N. I. Polzikova, D. V. Kuznetsov, and L. Schimansky-Geier, J. Appl. Phys. 90, 5425 (2001); I. E. Dikshtein, D. V. Kuznetsov, and L. Schimansky-Geier, Phys. Rev. E 65, 061101 (2002).

[4] K. L. Sebastian and A. K. R. Paul, Phys. Rev. E 62, 927 (2000).

[5] S. K. Lee and W. Sung, Phys. Rev. E 63, 021115 (2001); 64, 041801 (2001).

[6] P. Kraikivsky, R. Lipowsky, and J. Kiefeld, Europhys. Lett. 66, 763 (2004); Eur. Phys. J. E 16, 319 (2005).

[7] D. Hennig, L. Schimansky-Geier, and P. Hänggi, Europhys. Lett. 78, 20002 (2007); D. Hennig, S. Fugmann, L. SchimanskyGeier, and P. Hänggi, Phys. Rev. E 76, 041110 (2007); S. Fugmann, D. Hennig, L. Schimansky-Geier, and P. Hänggi, ibid. 77, 061135 (2008).
[8] P. Maniadis and S. Flach, Europhys. Lett. 74, 452 (2006).

[9] J. L. Marin, F. Falo, P. J. Martinez, and L. M. Floria, Phys. Rev. E 63, 066603 (2001).

[10] P. J. Martinez, M. Meister, L. M. Floria, and F. Falo, Chaos 13, 610 (2003).

[11] D. Hennig, L. Schimansky-Geier, and P. Hänggi, Europhys. Lett. 83, 60008 (2008).

[12] R. Khomeriki, S. Lepri, and S. Ruffo, Phys. Rev. E 64, 056606 (2001); Physica D 168, 152 (2002).

[13] T. Cretegny, T. Dauxois, S. Ruffo, and A. Torcini, Physica D 121, 109 (1998).

[14] G. P. Tsironis and S. Aubry, Phys. Rev. Lett. 77, 5225 (1996).

[15] A. Bikaki, N. K. Voulgarakis, S. Aubry, and G. P. Tsironis, Phys. Rev. E 59, 1234 (1999).

[16] R. Reigada, A. H. Romero, A. Sarmiento, and K. Lindenberg, J. Chem. Phys. 111, 1373 (1999). 